\title{
On a Cumulative Distribution Function Related to the Bernoulli Process
}

\author{
Peter Kopanov $^{1, *}$, Miroslav Marinov ${ }^{2}$ \\ ${ }^{1}$ Department of Mathematics and Informatics, Plovdiv University "Paisii Hilendarski", 4000, Plovdiv, Bulgaria \\ ${ }^{2}$ St Catherine's College, Oxford University, OX1 3UJ, Oxford, United Kingdom
}

Copyright $@ 2017$ by authors, all rights reserved. Authors agree that this article remains permanently open access under the terms of the Creative Commons Attribution License 4.0 International License

\begin{abstract}
We examine the properties of a cumulative distribution function which is related to the Bernoulli process. Results figuring in a paper ${ }^{[1]}$ are shown and new ones are included. Most of them are connected to the behaviour of the probability density function (derivative) of the given distribution.
\end{abstract}

Keywords Bernoulli Process, Density Function, Criterion

\section{Introduction}

A Bernoulli process is a sequence (finite or infinite) of independent random variables $a_{1}, a_{2}, a_{3}, \ldots$, such that for each $i$ :

- The value of $a_{i}$ is either 0 or 1

- The probability of the event $a_{i}=1$ is a real constant $p \in[0,1]$ (respectively, the probability of $a_{i}=0$ is $1-p)$.

Let $\Omega$ be the set of all infinite binary sequences. If we consider an infinite Bernoulli process, a specific outcome $\omega$ will belong to $\Omega$. It is known that for all $\omega \in \Omega$ the probability $\omega$ occurs as a result of such a process is 0 .

Now let $X \in[0,1]$ be a random variable, such that for each $\omega=a_{1}, a_{2}, \ldots$ the value $X(\omega)$ is the fraction $0 . a_{1} a_{2} \ldots$ For example, the outcome $\omega=1,0,0,1,0,0, \ldots$ corresponds to the binary $X(\omega)=0.1001$ (which in decimal system equals $0.5625)$. Obviously $X(\omega)$ depends on $p$.

For each $x \in \mathbb{R}$ by $F_{p}(x)$ we will represent the probability $\mathbb{P}[X(\omega) \leq x]$ (the cumulative distribution function of $X$ ). We have $F_{p}(x)=0$ for all $x<0$ and $F_{p}(x)=1$ for all $x>1$. The interesting domain is $[0,1]$.
Note that, as any distribution function ${ }^{[3]}, F_{p}(x)$ is nondecreasing and right-continuous in $[0,1]$.

In the following section we shall cite properties of this function, some of which describe it as a singular function. As such it is natural to consider $F_{p}(x)$ 's formal derivative - the probability density function $f_{p}(x)=F_{p}^{\prime}(x)$ in $x \in[0,1]$ (there are only partial results ${ }^{[1]}$ on it). We will derive new results on it in the rest of the paper.

\section{Basic properties}

On Figure 1 are shown different approximation (except $p=\frac{1}{2}$ ) graphs of $F_{p}(x)$, all of which depend on the value of $p$ (as $p$ increases, the $F_{p}(x)$-value for a given $x$ decreases). We shall omit the trivial cases $p=0$ and $p=1$. If $p=\frac{1}{2}$ the graph lies on the line $y=x$. Indeed, here $\mathbb{P}\left[a_{i}=1\right]=P\left[a_{i}=0\right]$ thus $F_{p}(x)$ equals the probability a random number in $[0,1]$ is less than or equal to a fixed number $x$ (which is exactly $x$ ). The arc length when $p=\frac{1}{2}$ equals $\sqrt{2}$.

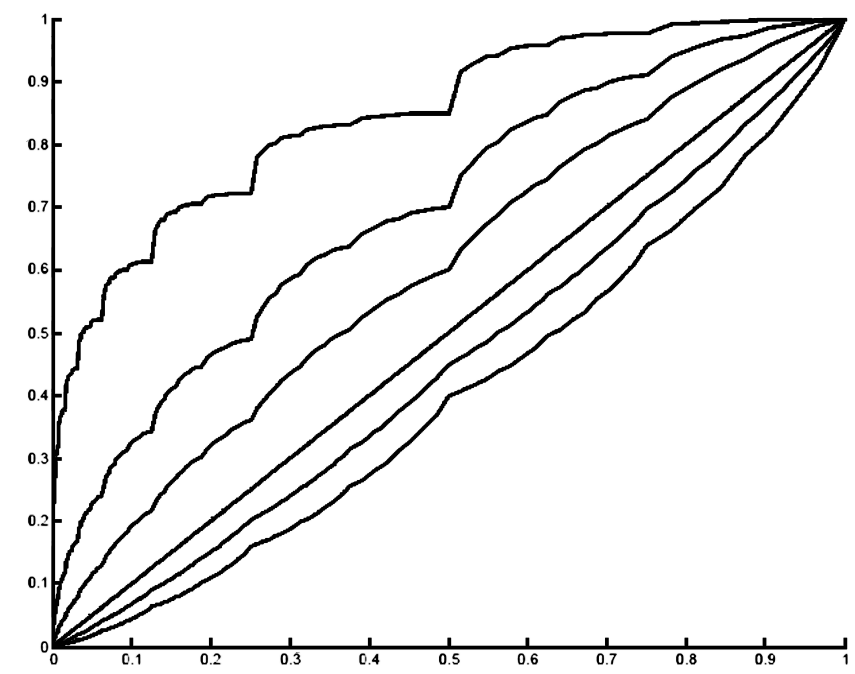

Figure 1. Different graphs of $F_{p}(x)$ 
For the non-trivial cases the graphs show that the function is strictly increasing and continuous. Its Riemann integral in $[0,1]$ equals $1-p$. For the arc length there is an interesting result - it always equals 2 regardless the value of $p$. The Triangle inequality implies that if we divide the graph into infinitely small partitions, each one is either parallel to the $x$ or to the $y$-axis. All these statements have already been proven $^{[1]}$.

\section{Useful functional equations}

Set $x=\sum_{i=1}^{\infty} \frac{a_{i}}{2^{i}}$. We have the following equalities ${ }^{[1]}$ :

Proposition 1.1. $F_{p}\left(\frac{x}{2}\right)=(1-p) F_{p}(x)$

Proposition 1.2. $F_{p}\left(\frac{x+1}{2}\right)=1-p+p F_{p}(x)$

Proposition 2. $F_{p}(x)=1-F_{1-p}(1-x)$

From the first two propositions we can directly deduce that

Proposition 3. $F_{p}\left(0 . a_{1} a_{2} \ldots\right)=(1-p) a_{1}+(1-p+(2 p-$ 1) $\left.a_{1}\right) F_{p}\left(0 . a_{2} a_{3} \ldots\right)$

This equation is suitable for creating a recursive algorithm for calculating $F_{p}(x)$ for an arbitrary $x \in[0,1]$ (the process may be an approximation with a negligible error if $x$ is non-dyadic).

\section{The derivative (density) of the cumulative function}

We shall omit the case $p=\frac{1}{2}$, where clearly $f_{p}(x)=1$ in $x \in(0,1)$.

\subsection{Basic facts and useful lemmas}

In Section 1 we mentioned the infinite staircase behaviour of $F_{p}(x)^{\prime}$ s graph. It directly implies that in each point in $[0,1]$ the derivative $f_{p}(x)=F_{p}^{\prime}(x)$ of our function is either 0 , $+\infty$ or does not exist. Moreover, since $F_{p}(x)$ is a monotonic (strictly increasing) function, due to Lebesgue's theorem for monotonic functions ${ }^{[4]}$ its derivative exists almost everywhere in $[0,1]$. So how does the density function exactly behave - how can we describe, depending on $p$, which are the points in which it is defined? We shall derive a criterion which answers the previous question.

Firstly, we have the following:

Lemma 4.1. If $f_{p}(x)$ exists then $f_{p}(x)=f_{1-p}(1-x)$. Proof. Differentiating both sides of the equation in Proposition 2 with respect to $x$ gives

$$
\begin{gathered}
f_{p}(x)=\frac{d F_{p}(x)}{d x}=-\frac{d F_{1-p}(1-x)}{d x}= \\
=-\frac{d F_{1-p}(1-x)}{d(1-x)} \frac{d(1-x)}{d x}=\frac{d F_{1-p}(1-x)}{d(1-x)}=f_{1-p}(1-x)
\end{gathered}
$$

as desired.

Remark. In particular, the upper equality also holds for the left and right-hand derivatives of $F_{p}(x)$ and $F_{1-p}(1-x)$.

It would be suitable to have an expression of the form $F_{p}(x+h)-F_{p}(x)$ for an appropriate $h$ (in this case: $\left.h=\frac{1}{2^{n}}\right)$. Then using that $f_{p}(x)=0$ or $+\infty$ when it exists, a squeeze-type technique (using this expression) will be sufficient to describe its behaviour in a given point.

Set $s_{n}(x)=\sum_{i=1}^{n} a_{i}$ (i.e. $s_{n}(x)$ represents the number of 1's among the first $n$ bits in $x$ 's binary representation). Then:

Lemma 4.2. For every $n \in \mathbb{N}$ we have:

$$
\begin{gathered}
F_{p}\left(0 . a_{1} \ldots a_{n-1} 1 a_{n+1} \ldots\right)-F_{p}\left(0 . a_{1} \ldots a_{n-1} 0 a_{n+1} \ldots\right)= \\
=p^{s_{n}(x)}(1-p)^{n-s_{n}(x)}\left(1+\frac{2 p-1}{1-p} F_{p}\left(y_{n}\right)\right)
\end{gathered}
$$

where $x=0 . a_{1} \ldots a_{n-1} 0 a_{n+1} \ldots$ and $y_{n}=0 . a_{n+1} a_{n+2} \ldots$.

Proof. Apply Proposition 3. $n-1$ times (the $i$-th for $\left.F_{p}\left(0 . a_{i} a_{i+1} \ldots\right)\right)$, then:

$F_{p}\left(0 . a_{1} a_{2} \ldots 1 \ldots\right)-F_{p}\left(0 . a_{1} a_{2} \ldots 0 \ldots\right)=$

$=(1-p) a_{1}+\left(1-p+(2 p-1) a_{1}\right)\left((1-p) a_{2}+(1-p+\right.$ $\left.(2 p-1) a_{2}\right)\left((1-p) a_{3}+\left(1-p+(2 p-1) a_{3}\right)(\ldots((1-\right.$ p) $\left.\left.a_{n-1}+\left(1-p+(2 p-1) a_{n-1}\right) F_{p}\left(0.1 a_{n+1} \ldots\right)\right) \ldots\right)$

$-(1-p) a_{1}-\left(1-p+(2 p-1) a_{1}\right)\left((1-p) a_{2}+(1-p+\right.$ $\left.(2 p-1) a_{2}\right)\left((1-p) a_{3}+\left(1-p+(2 p-1) a_{3}\right)(\ldots((1-\right.$ p) $\left.\left.a_{n-1}+\left(1-p+(2 p-1) a_{n-1}\right) F_{p}\left(0.0 a_{n+1} \ldots\right)\right) \ldots\right)$

Now we can firstly eliminate $(1-p) a_{1}$ and $-(1-p) a_{1}$. After that we take out in front of the brackets $\left(1-p+(2 p-1) a_{1}\right)$. In the brackets we eliminate $(1-p) a_{2}$ and $-(1-p) a_{2}$, then we take out $\left(1-p+(2 p-1) a_{2}\right)$ to the front and so on (this occurs $n-1$ times). We reach:

$$
\begin{gathered}
F_{p}\left(0 . a_{1} a_{2} \ldots 1 \ldots\right)-F_{p}\left(0 . a_{1} a_{2} \ldots 0 \ldots\right)= \\
=\prod_{i=1}^{n-1}\left(1-p+(2 p-1) a_{i}\right) .\left(F_{p}\left(0.1 a_{n+1} \ldots\right)-F_{p}\left(0.0 a_{n+1} \ldots\right)\right)
\end{gathered}
$$

Now if $a_{i}=0$, the $i$-th multiplier in the upper expression equals $1-p$, else it equals $p$. Therefore:

$$
\prod_{i=1}^{n-1}\left(1-p+(2 p-1) a_{i}\right)=p^{s}(1-p)^{n-s-1}
$$

On the other hand, Propositions 1.1. and 1.2. give:

$$
\begin{gathered}
F_{p}\left(0.1 a_{n+1} \ldots\right)-F_{p}\left(0.0 a_{n+1} \ldots\right)= \\
1-p+p F_{p}\left(y_{n}\right)-(1-p) F_{p}\left(y_{n}\right)= \\
=1-p+(2 p-1) F_{p}\left(y_{n}\right)=(1-p)\left(1+\frac{2 p-1}{1-p} F_{p}\left(y_{n}\right)\right)
\end{gathered}
$$

Multiplying the last two equalities gives the result.

Remark 4.3. For simplicity, let $A\left(y_{n}\right)=\left(1+\frac{2 p-1}{1-p} F_{p}\left(y_{n}\right)\right)$. Note that $A\left(y_{n}\right)$ is bounded and always positive. The first is because $p=$ const and $f_{p}(y) \in[0,1]$ - for $p \in\left(\frac{1}{2}, 1\right)$ we have $1 \leq A\left(y_{n}\right) \leq 1+\frac{2 p-1}{1-p}$, and if $p \in\left(0, \frac{1}{2}\right)$ then $1+\frac{2 p-1}{1-p} \leq A\left(y_{n}\right) \leq 1$. The second follows from $1+\frac{2 p-1}{1-p}=\frac{p}{1-p}>0$ (referring to the previous double inequalities). 


\subsection{The special dyadic case}

An important characteristic property of the dyadic numbers is that the value $s_{n}(x)$ is finite for all $n$ - that is due to the fact that from a bit onwards there are only 0's. Set $\lim _{n \rightarrow \infty} s_{n}(x)=s_{\infty}(x)$. We shall prove that:

Theorem 5. Let $x \in[0,1]$ be a dyadic number. Then $f_{p}(x)$ does not exist.

Proof. It suffices to show that as $h \rightarrow 0$ the limit of $\frac{F_{p}(x+h)-F_{p}(x)}{h}$ is different in the cases $h= \pm \frac{1}{2^{n}}$. Consider the limit in the case $h=\frac{1}{2^{n}}$ (denote it by $\left.C_{p}(x)\right)$. We have:

$$
\begin{gathered}
C_{p}(x)=\lim _{n \rightarrow \infty}\left[2^{n} p^{s_{n}(x)}(1-p)^{n-s_{n}(x)} A\left(y_{n}\right)\right]= \\
=\left(\frac{p}{1-p}\right)^{s_{\infty}(x)} \lim _{n \rightarrow \infty}\left[(2(1-p))^{n} A\left(y_{n}\right)\right]
\end{gathered}
$$

Neglecting the positive $\left(\frac{p}{1-p}\right)^{s_{\infty}(x)}$ and the bounded multiplier $A\left(y_{n}\right)$, depending on whether $2(1-p)$ is less or greater than 1 (recall that $p \neq \frac{1}{2}$ ), we deduce that:

$$
C_{p}(x)= \begin{cases}0 & \text { if } p>\frac{1}{2} \\ +\infty & \text { if } p<\frac{1}{2}\end{cases}
$$

However, if $D_{p}(x)$ is the same limit, but with $h=-\frac{1}{2^{n}}$, then by the remark in Lemma 4.1 $D_{p}(x)=C_{1-p}(1-x)$, so since $1-x$ is also a dyadic number, we have:

$$
D_{p}(x)= \begin{cases}0 & \text { if } p<\frac{1}{2} \\ +\infty & \text { if } p>\frac{1}{2}\end{cases}
$$

We conclude that $C_{p}(x) \neq D_{p}(x)$ and the proof is completed.

\subsection{The criterion and examples}

The criterion will mainly rely on Lemma 4.2. Since the expression there contains a combination of $s_{n}(x)$ and $n$, it would be convenient to use the notation $k_{n}(x)=\frac{s_{n}(x)}{n}$ (i.e. $k_{n}(x)$ is the density of the 1-bits among the first $\left.n\right)$. Also, set $k(x)=\lim _{n \rightarrow \infty} k_{n}(x)$ (if this limit exists) and $t=p-0.5$ the oriented distance from $p$ to 0.5 on the real line.

Theorem 6. A Criterion for the behaviour of $f_{p}(x)$ in almost all real $x \in[0,1]$

For all real $x \in[0,1]$ for which $k(x)$ exists and $k(x) \neq 0,1$ we have

$f_{p}(x)= \begin{cases}0, & \text { if }(1+2 t)^{k(x)}(1-2 t)^{1-k(x)}<1 \\ +\infty, & \text { if }(1+2 t)^{k(x)}(1-2 t)^{1-k(x)}>1 \\ \text { does not exist, }, & \text { if }(1+2 t)^{k(x)}(1-2 t)^{1-k(x)}=1\end{cases}$

Proof. Consider $\frac{1}{2^{n+1}} \leq h \leq \frac{1}{2^{n}}$. Then since $F_{p}(x)$ is strictly increasing, we have:

$$
\frac{F_{p}\left(x+\frac{1}{2^{n+1}}\right)-F_{p}(x)}{\frac{1}{2^{n}}} \leq \frac{F_{p}(x+h)-F_{p}(x)}{h} \leq
$$

$$
\frac{F_{p}\left(x+\frac{1}{2^{n}}\right)-F_{p}(x)}{\frac{1}{2^{n+1}}}
$$

hence

$$
\begin{gathered}
\lim _{n \rightarrow \infty}\left[\frac{F_{p}\left(x+\frac{1}{2^{n+1}}\right)-F_{p}(x)}{\frac{1}{2^{n}}}\right] \leq f_{p}(x) \\
\leq \lim _{n \rightarrow \infty}\left[\frac{F_{p}\left(x+\frac{1}{2^{n}}\right)-F_{p}(x)}{\frac{1}{2^{n+1}}}\right]
\end{gathered}
$$

The left-hand and right-hand sides are respectively equal to (note that $k(x)(1-k(x)) \neq 0$ )

$$
\begin{gathered}
L_{p}(x)=\lim _{n \rightarrow \infty}\left[2^{n} p^{s_{n+1}(x)}(1-p)^{n+1-s_{n+1}(x)} A\left(y_{n+1}\right)\right]= \\
\quad=\lim _{n \rightarrow \infty}\left[\frac{1}{2}\left((1+2 t)^{k(x)}(1-2 t)^{1-k(x)}\right)^{n+1} A\left(y_{n+1}\right)\right]
\end{gathered}
$$

and (analogously)

$$
R_{p}(x)=\lim _{n \rightarrow \infty}\left[2\left((1+2 t)^{k(x)}(1-2 t)^{1-k(x)}\right)^{n} A\left(y_{n}\right)\right]
$$

As $t$ and $x$ vary, we have the following cases for the multiplier $(1+2 t)^{k(x)}(1-2 t)^{1-k(x)}$ :

1. If it equals 1 , then consider $f_{p}(x)$ again with $h=\frac{1}{2^{n}}$. We analogously have:

$$
\begin{gathered}
C_{p}(x)=\lim _{n \rightarrow \infty}\left[\left((1+2 t)^{k(x)}(1-2 t)^{1-k(x)}\right)^{n} A\left(y_{n}\right)\right]= \\
=\lim _{n \rightarrow \infty} A\left(y_{n}\right)
\end{gathered}
$$

Here everything depends on $A\left(y_{n}\right)$, in particular - on $F_{p}\left(y_{n}\right)$. We shall prove that $F_{p}\left(y_{n}\right)$ oscillates around $F_{p}\left(\frac{1}{2}\right)=1-p$ but does not tend to it. If from a bit onwards in $y$ 's binary expansion there are only $1 \mathrm{~s}$, then $y$ is actually a dyadic, but this case has already been examined. Hence we can assume that $y$ is not dyadic.

Depending on $n$, the $(n+1)$-th bit (after the decimal point) will be either 1 or 0 . We have an infinite alternation (not necessarily periodic) of these two possibilities. The two cases respectively give $y>\frac{1}{2}$ and $y<\frac{1}{2}$. Therefore, to have an existing limit, we need $y$ to tend (from left and right) to $\frac{1}{2}$. In order to happen from the right side, we need from a bit onwards to have: if it is 1 , the next one is 0 (else $0.11 \ldots>\frac{3}{4}$ ). Conversely, from the left side we need from a bit onwards: if this bit is 0 , then the next one is 1 . Combining the two necessities, we have (from a bit onwards) either $y=0 .(01)$ or $y=0 .(10)$, respectively $\frac{1}{3}<\frac{1}{2}$ and $\frac{2}{3}>\frac{1}{2}$, a contradiction.

Hence the limit $C_{p}(x)$ does not exist and so does the derivative.

2. If it is strictly less than 1 , then the bounded multiplier $A\left(y_{n}\right)>0$ affects nothing - we have $L_{p}(x)=R_{p}(x)=$ 0 , therefore $f_{p}(x)=0$.

3. If it is strictly greater than 1 , then the bounded multiplier $A\left(y_{n}\right)>0$ affects nothing - we have $L_{p}(x)=R_{p}(x)=$ $+\infty$, therefore $f_{p}(x)=+\infty$. 
The proof is completed.

Remark. We have considered $k(x) \neq 0,1$ because $\lim _{n \rightarrow \infty}\left((1+2 t)^{k_{n}(x)}\right)^{n+1}=\lim _{n \rightarrow \infty}\left((1+2 t)^{k(x)}\right)^{n+1}$ is not necessarily true if $k(x)=0$ ( similarly for $1-k(x)=0$ ).

Our criterion is ready and we can test it. The following example illustrates the measure one subset of $[0,1]$, in which the derivative is 0 for all non-trivial $p$. Indeed, it is proven ${ }^{[2]}$ that the subset of real numbers with $k(x)=\frac{1}{2}$ (the so-called Regular numbers) has Lebesgue measure 1 in $[0,1]$. Now:

Theorem 7. Let $k \in(0,1)$ and consider the set of all real numbers $x$ with limit density value $k(x)=k$. Then for all non-trivial $p$, it holds that $f_{p}(x)=0$ at all numbers in this set if and only if $k=\frac{1}{2}$.

Proof. Due to Theorem 6. we actually have to prove that: The inequality $(1+2 t)^{k}(1-2 t)^{1-k}<1$ holds for all $t \in(-0.5,0) \cup(0,0.5)$ if and only if $k=\frac{1}{2}$.

$(\Leftarrow)$ If $k=\frac{1}{2}$, then we have $\sqrt{1-4 t^{2}}<1$. The last holds for all given $t$.

$(\Rightarrow)$ The given inequality can be rewritten as $\left(1-4 t^{2}\right)^{\frac{1}{2}}<\left(\frac{1-2 t}{1+2 t}\right)^{k-\frac{1}{2}}$.

Denote the left-hand and right-hand sides by $f(t)$ and $g(t)$, respectively. Note that $f(0)=g(0)=1$ and that $f(t)$ attains its maximum at $t=0$. Hence if $f(t)$ and $g(t)$ intersect at $t=0$, then there will be a region where $g(t)<f(t)$, a contradiction. Therefore, a necessary condition for the inequality to hold is $-f$ and $g$ are tangent at $t=0$. But then $f^{\prime}(0)=g^{\prime}(0)$ and since $f^{\prime}(0)=0$, we finally have:

$$
\begin{gathered}
g^{\prime}(t)=\left(k-\frac{1}{2}\right)\left(\frac{1-2 t}{1+2 t}\right)^{-k-\frac{1}{2}} *\left(\frac{-4}{(1+2 t)^{2}}\right) \Leftrightarrow \\
0=g^{\prime}(0)=2-4 k \Leftrightarrow k=\frac{1}{2}
\end{gathered}
$$

which completes the proof.

The last two theorems show that for all non-trivial $p$ there exist sets (of measure 0 over $\mathbb{R}$ ) in which $f_{p}(x)$ does not exist. All these are interesting only from the constructive point of view (but not from the probabilistic because their measure is 0 ). Let us show an example.

Proposition 8. For all $l \in \mathbb{N}$ :

$$
f_{p}\left(\frac{6}{7.2^{l}}\right) \begin{cases}=0, & p \in\left(0, \frac{1}{2}\right) \cup\left(\frac{\sqrt{5}+1}{4}, 1\right) \\ +\infty, & p \in\left(\frac{1}{2}, \frac{\sqrt{5}+1}{4}\right) \\ \text { does not exist, } & p=\frac{\sqrt{5}+1}{4}\end{cases}
$$

Proof. Note that all such numbers have a binary expansion $0.00 . .0(110)$, hence they have limit density $k=\frac{2}{3}$ and the derivative depends on the expression $(1+2 t)^{\frac{2}{3}}(1-2 t)^{\frac{1}{3}}$. With Wolfram Mathematica we can check that the last is greater than or equal to 1 when $t \in\left(0, \frac{\sqrt{5}-1}{4}\right]$ and less than 1 in the other cases. Finally, $p=0.5+t$ gives the result.

\subsection{The derivative when $k_{n}(x)$ diverges}

In this case both of the non-constant multipliers in the expressions for $L_{p}(x)$ and $R_{p}(x)$ diverge, too. This, in general, does not exclude the possibility that their product converges. The author still does not have any results about such cases.

\section{REFERENCES}

[1] Cohen M., Nater J., Wear P., Tossing a coin, 18.821 Project Laboratory in Mathematics (2013)

[2] Ross S., A First Course in Probability, California, Pearson, 8-th edition (2010), Chapter 4, p.168

[3] Lamperti J., Probability, New York, A survey of the mathematica theory, (1966), p.39, Theorem 2.

[4] Riss F., Lecons D'Analyse Fonctionnelle, Budapest, Academiai Kiado, Sixteme edition (1972), p.15 